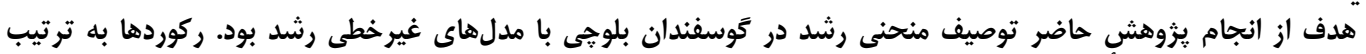

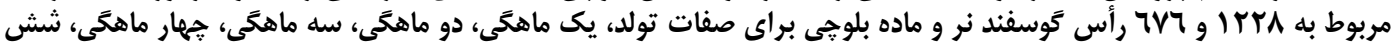

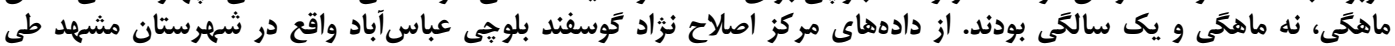

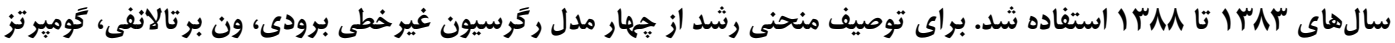

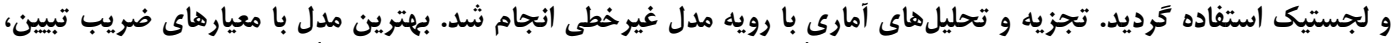

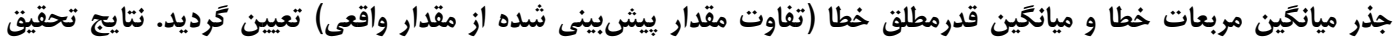

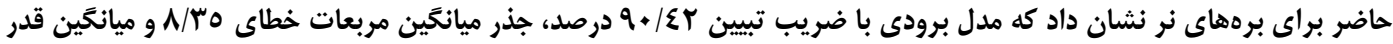

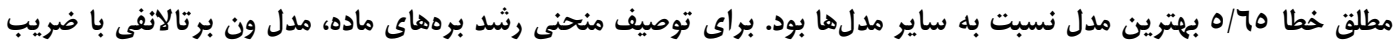

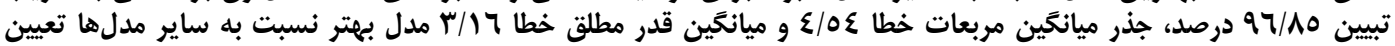

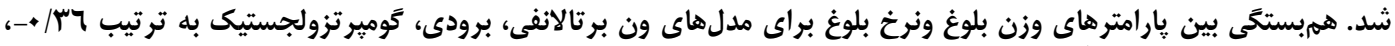

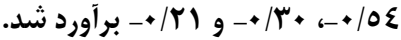

وازههاى كليدى: تَوسفند، برودى، ون بر تالانفى، كَوميرتز، لجستيك

كوسفندان بوده و تحت تأثير گونه، نزاد، مديريت، محيط و

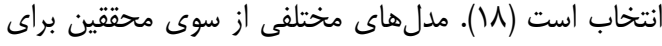

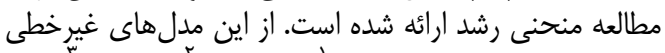

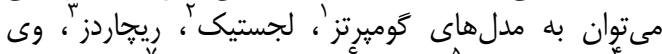

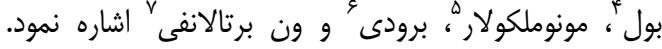

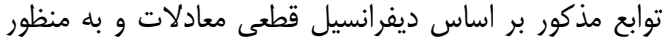

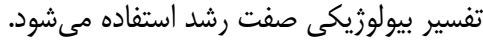

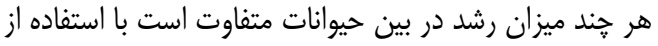

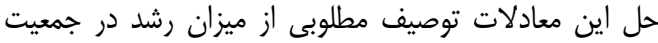

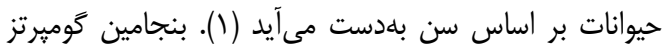

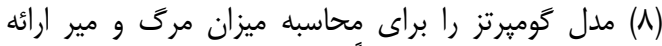

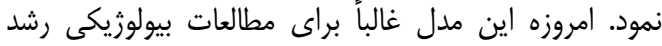

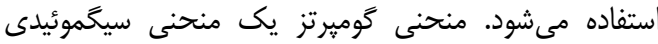

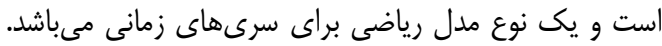

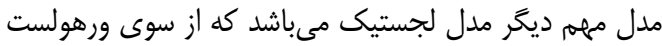
(19) براى صفت رشد ارائه شد. در اين تابع نقطه عطف

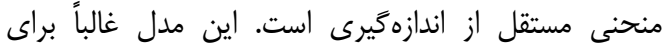

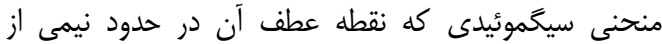

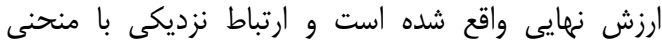

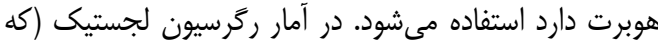

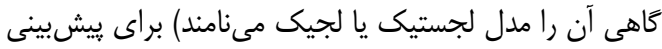

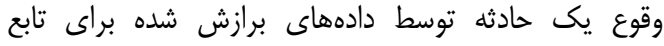

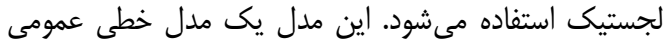

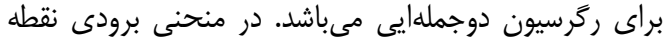

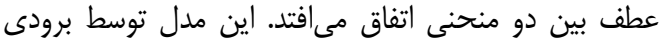

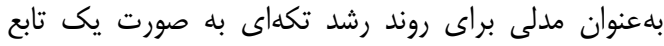

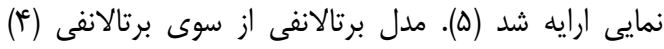

صفت رشد يكى از مهمترين صفات ضرورى برى براى

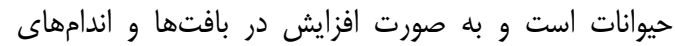

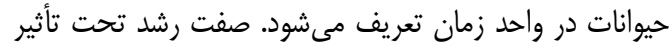

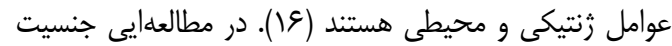

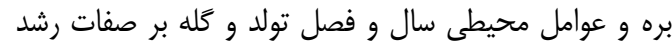

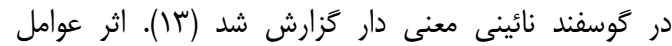

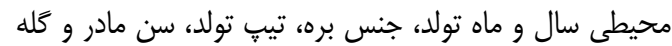

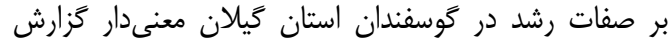

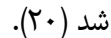

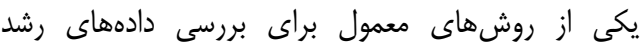

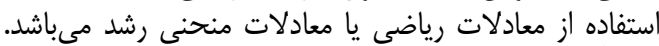

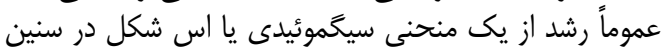

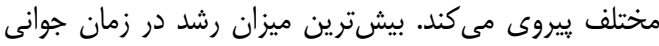

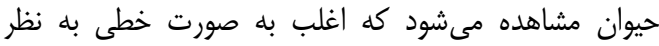

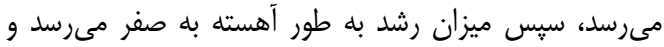

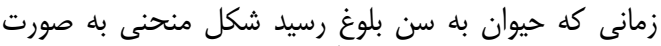

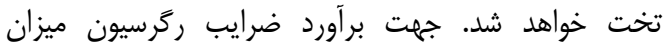

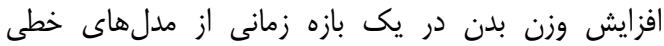

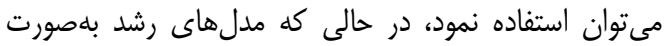

غيرخطى هستند (1) (1). از جمله راههايى كه مىتوان اثرات مختلف رانف را روى عملكرد

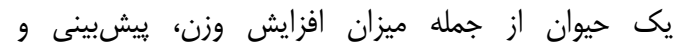

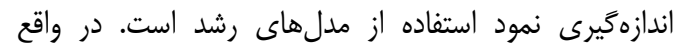

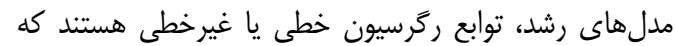

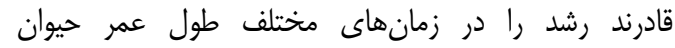

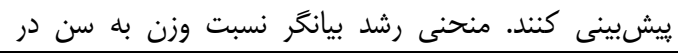

3- Richards 4- Wei bull


$\mathrm{W}_{\mathrm{t}}=\mathrm{A} \exp \left(-\mathrm{Be}^{-\mathrm{Kt}}\right)$

$\mathrm{W}_{\mathrm{t}}=\mathrm{A} /\left(1+\mathrm{Be}^{-\mathrm{Kt}}\right)$

$\mathrm{W}_{\mathrm{t}}=\mathrm{A}\left(1-\mathrm{Be}^{-\mathrm{Kt}}\right)$

$\mathrm{W}_{\mathrm{t}}=\mathrm{A}\left(1-\mathrm{Be}^{-\mathrm{Kt}}\right)^{3}$

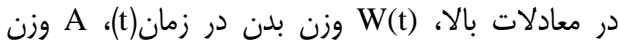

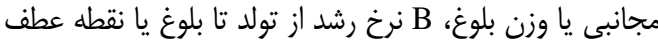

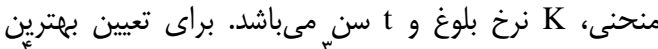

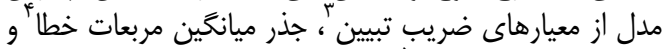

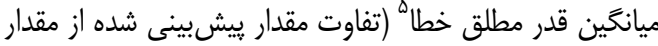

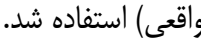

\section{نتايج و بحث}

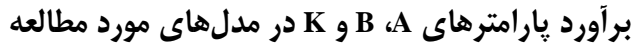

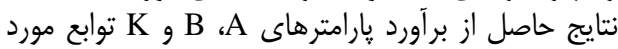

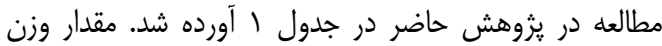

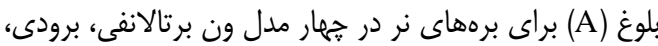

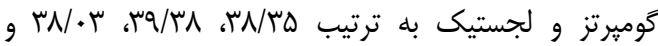

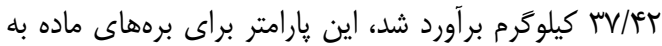

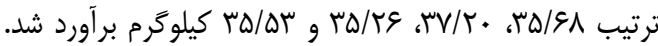

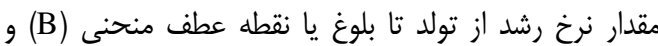

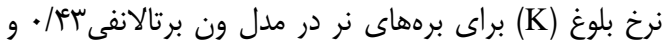

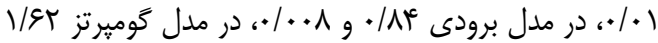

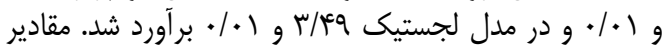

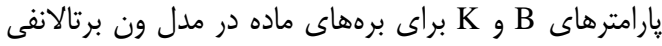

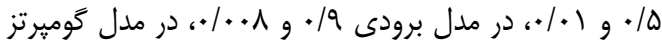

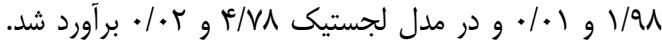

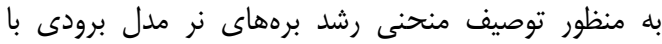

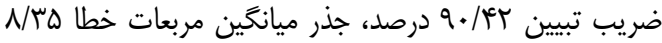

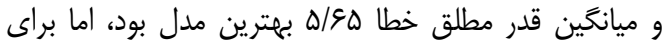

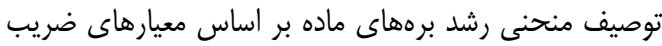

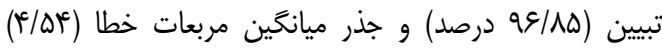

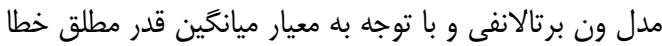

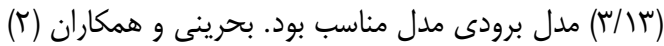

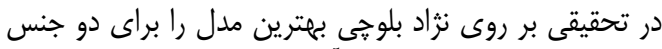

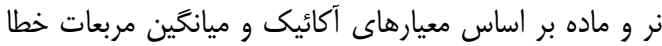

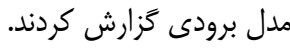

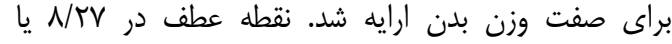

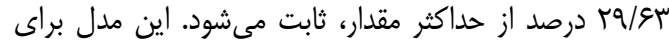

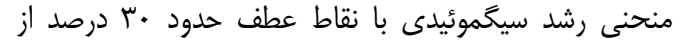

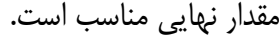

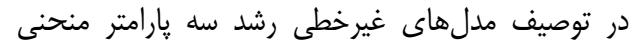

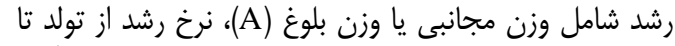

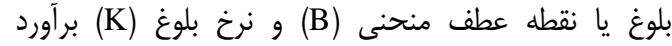

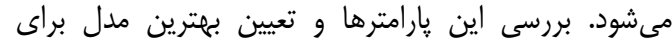

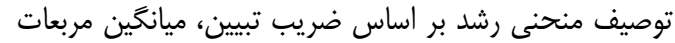

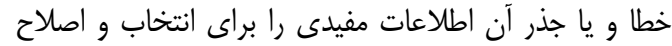

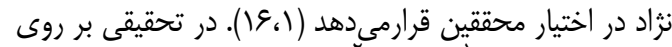

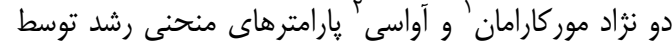

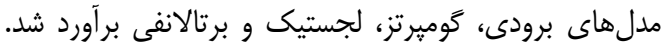

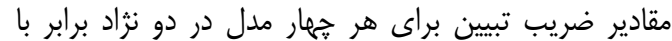

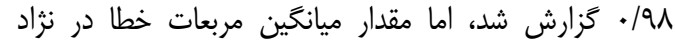

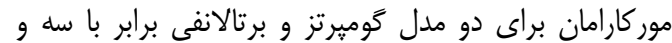

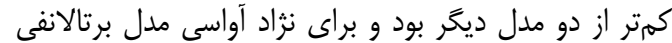

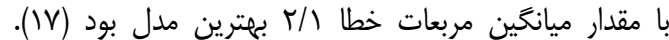

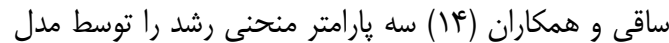

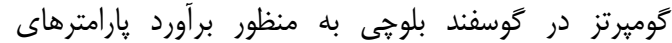
زنتيكى و فنوتيبى بر آورد نمودند.

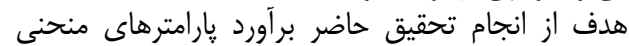

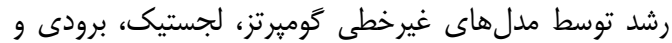

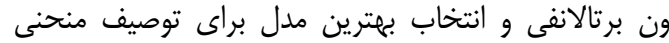
رشد در برهاى نر و ماده كوسفند بلوجى بود.

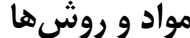

براى انجام تحقيق حاضر از دادهها مركز اصلاح ناد نزاد

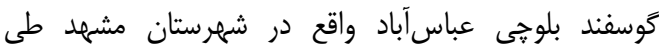

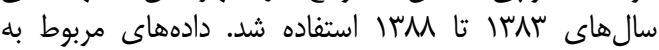

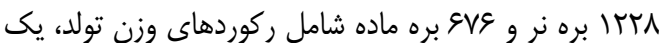

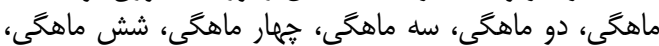

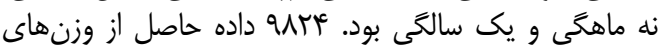

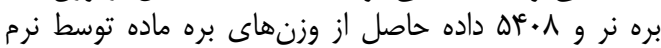

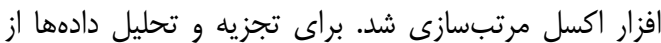

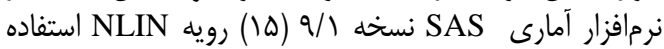

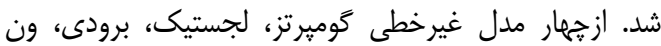
برتالانفى جهت توصيف منحنى رشد استفاده شئ كئ كه معادلات

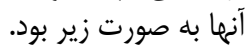

جدول ا- يارامترها و معيارهاى برآورد شده در دو جنس نر و ماده در مدلهاى مورد مطالعه Table 1. Estimated parameters and criteria in two sex of male and female in studied models

\begin{tabular}{|c|c|c|c|c|c|c|c|}
\hline MAE & RMSE & $\mathrm{R}^{2}$ & K & $\mathrm{B}$ & $\mathrm{A}(\mathrm{kg})$ & جنس & مدل \\
\hline$\Delta / V$ & N/FG & $9 . / 41$ & $\bullet / \bullet(\bullet / * \bullet r)$ & $\cdot / 4 \%(\cdot / . \bullet 9)$ & $r N / r \Delta(\cdot / \Gamma)$ & نر & \multirow{2}{*}{ ون برتالانفى } \\
\hline$r / 1 \varepsilon$ & $F / \Delta F$ & $৭ \& / \wedge \Delta$ & $\cdot / \cdot 1(\cdot / \cdot \cdots 1)$ & $\cdot / \Delta(\cdot / \cdots \Delta)$ & $r \Delta / \& \wedge(\cdot / \backslash Q)$ & ماده & \\
\hline$\Delta / \& \Delta$ & N/זם & $9 . / 45$ & $\cdot / \cdot \wedge(\cdot / \cdots r)$ & $\cdot \operatorname{l\Lambda f}(\cdot / . \cdot 9)$ & $r q / \mu \wedge(\cdot / r v)$ & نر & \multirow{2}{*}{ برودى } \\
\hline rו/ץ & $r / \Delta V$ & $q \cdot / 1 r$ & $\cdot / \cdot \wedge(\cdot / \cdots+)$ & $\cdot / 9(\cdot / \cdot+)$ & $r V / r(\cdot / 19)$ & ماده & \\
\hline$\Delta / N$ & N/F & $9 . / 4$. & $\bullet / .(\cdot / \cdots r)$ & V/GT $(\cdot / . T V)$ & $r N / \cdot r(\cdot / r \Lambda)$ & نر & \multirow{2}{*}{ كوميرتز } \\
\hline$r / r)$ & $r / \Delta S$ & $q \varepsilon / \wedge \mu$ & $\cdot / \cdot 1(\cdot / \cdot \cdot r)$ & $1 / Q \Lambda(\cdot / \cdot T \Delta)$ & $r \Delta / r q(\cdot / / F)$ & ماده & \\
\hline$\Delta / \Lambda$ & $\Lambda / \mu \Lambda$ & $9 . / r v$ & $\cdot / \cdot 1(\cdot / \cdot \cdots+r)$ & $r / f q(\cdot / 1)$ & $T V / F T(\cdot / T F)$ & نر & \multirow{2}{*}{ لجستيى } \\
\hline$r / \kappa$ & $r / 9 \Delta$ & $q \varepsilon / v$. & $\bullet \cdot r(\cdot / \cdot \cdots r)$ & $r / v \wedge(\cdot / 1)$ & $r \Delta / \Delta \Gamma(\cdot / I r)$ & ماده & \\
\hline
\end{tabular}

A: وزن بلوغ، B: نرخ رشد، K: نرخ بلوغ، R: ضريب تبيين، RMSE: جذر ميانكين مربعات خطا، MAE: ميانكَين قدر مطلق خطا، اعداد داخل يرانتز انحراف معيار مى باشد

1- Morkaraman

4- Root Mean Square Error (RMSE)
2- Awassi

5- Mean Absolute Error (MAE)
3- Coefficient of Determination $\left(\mathrm{R}^{2}\right)$ 


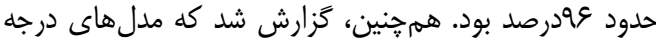

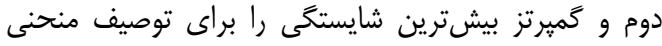

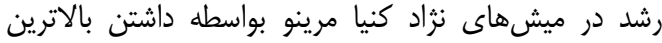

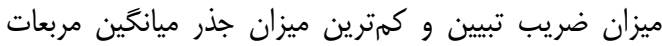

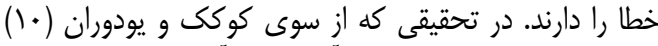

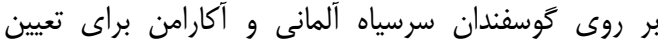

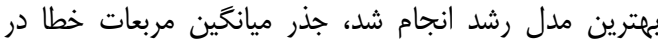

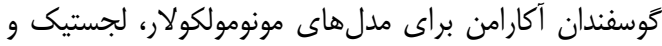

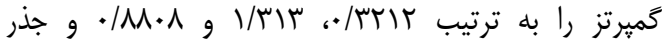

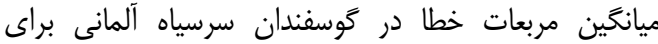

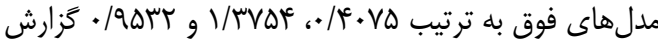

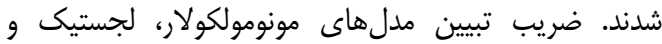

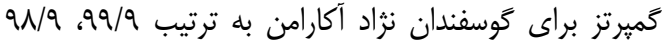

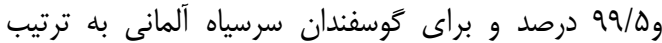

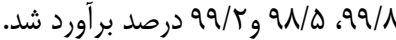

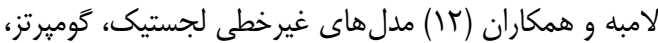

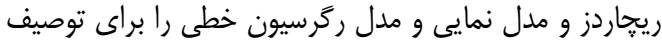

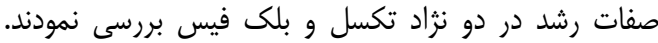

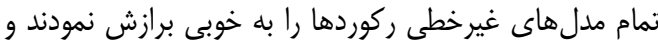

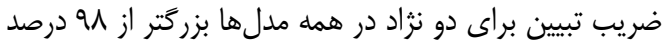

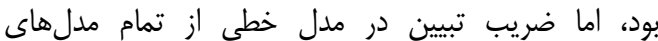

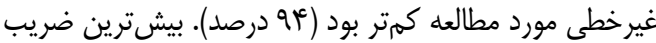

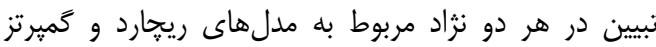

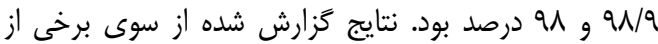

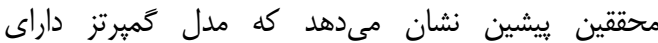

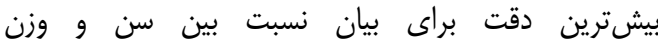

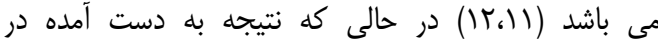

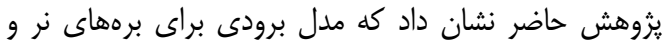

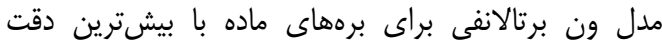

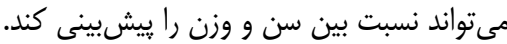

منحنى رشد برازش شده با مدلهاى ون برتان بالانفى،

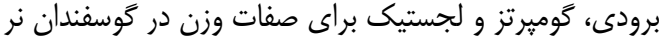

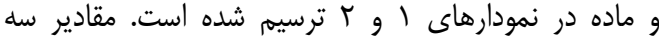

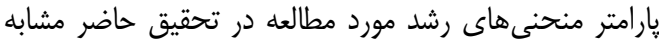

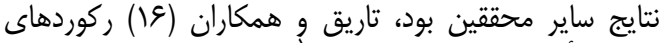

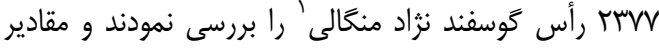

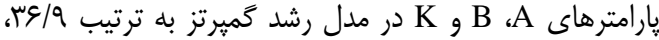

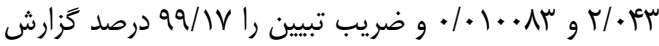

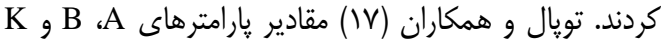

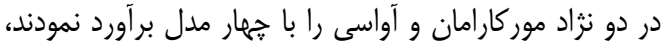

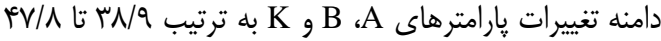

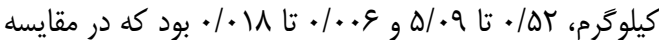

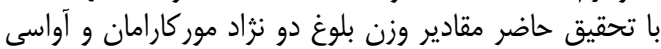

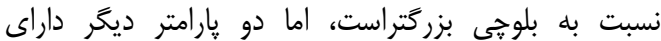
دامنهایى مشابهى مىباشند.

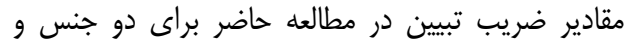

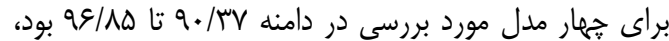

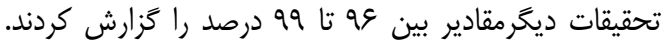

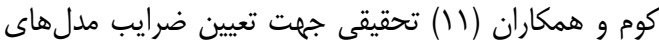

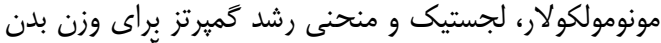

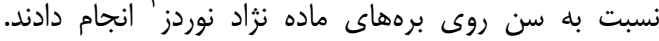

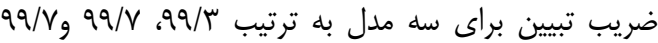

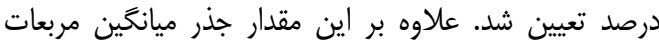

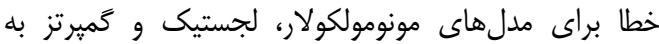

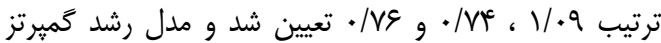

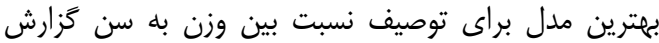

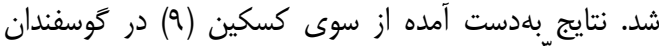

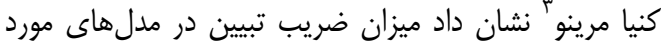

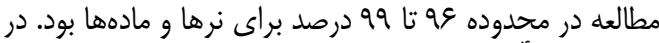

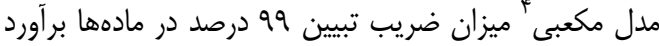
شد در حالى كه در مدل لجستيك اين ميزان كمتر بود ود و درد

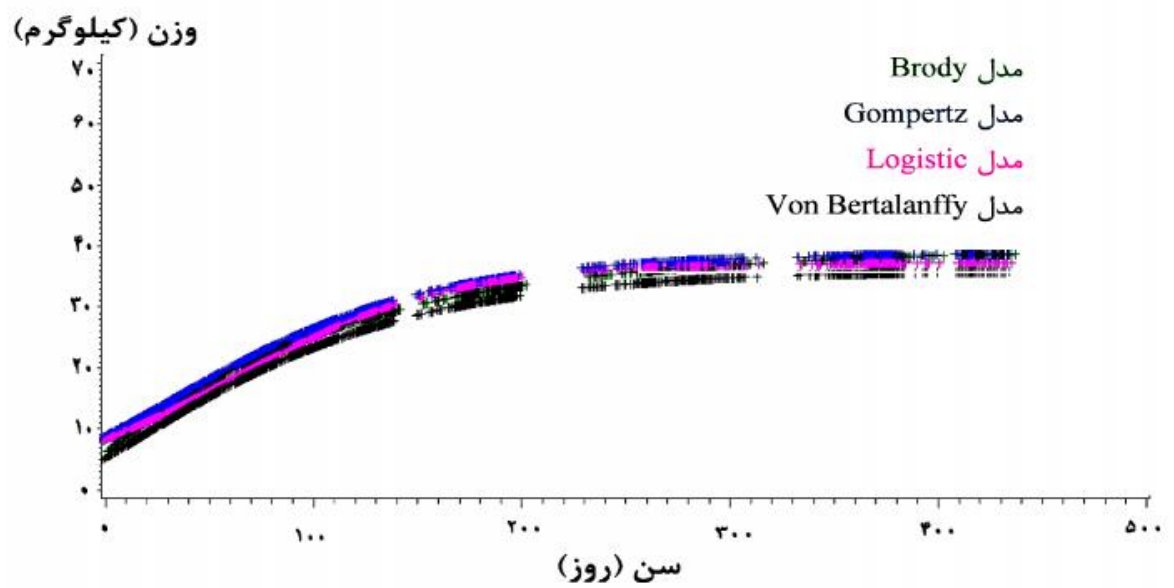

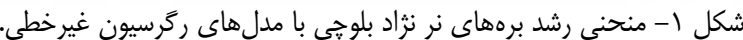

Figure 1. Growth curve of male lambs of Baluchi breed with nonlinear regression models. 


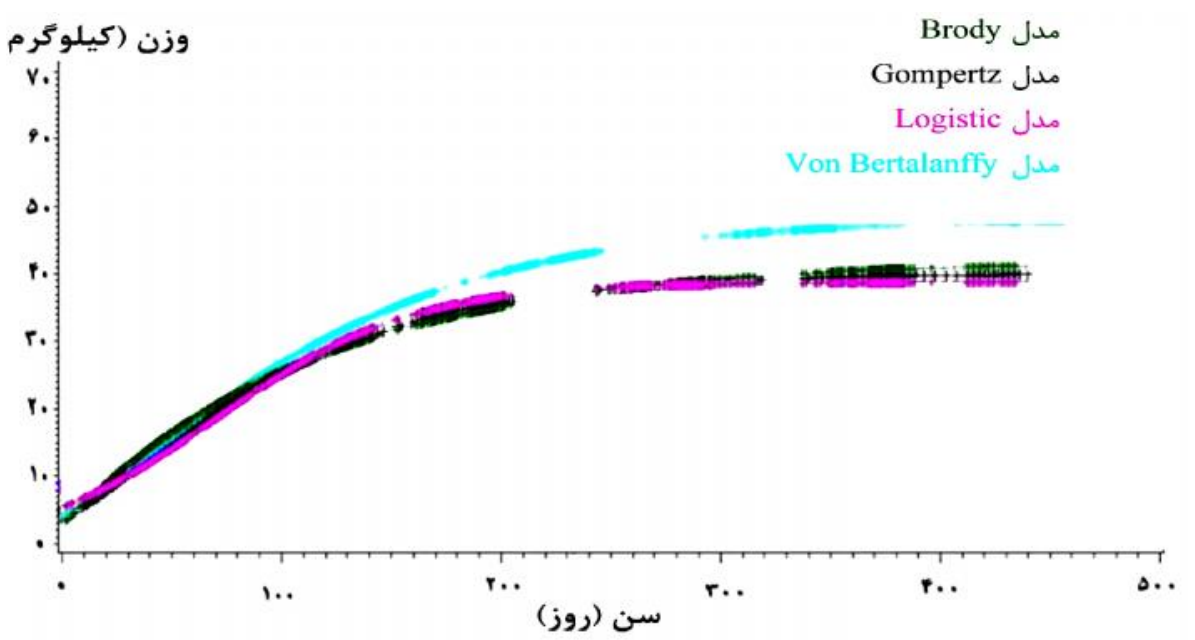

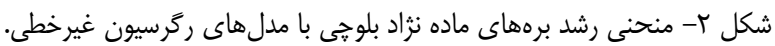

Figure 2. Growth curve of female lambs of Baluchi breed with nonlinear regression models

يارامترهاى A و K در همه مدلها منفى بلهدست آمد. اين

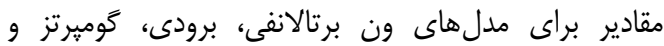

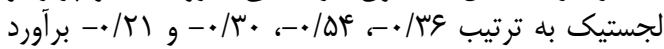

شد (1)
همبستخى بين يارامترهاى A، B و K در مدلهاى مورد

مطالعه همبستيكي

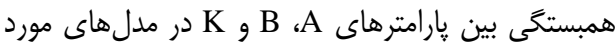

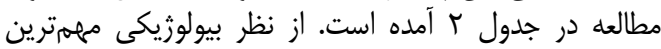

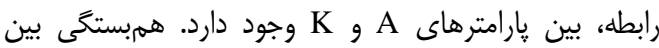

Table 2. Correlation between A, B and K parameters

جدول r- همبستخى بين يارامترهاى A B B

\begin{tabular}{|c|c|c|c|}
\hline $\mathrm{r}_{\mathrm{BK}}$ & $\mathrm{r}_{\mathrm{AK}}$ & $r_{A B}$ & مدل \\
\hline.$- / \cdot \Delta$ & $-\cdot /{ }^{\prime}$ & $-\cdot / / \mu$ & ون برتالانفى \\
\hline$-\cdot|\Delta|$ & $-\cdot / \Delta F^{2}$ & $\cdot / 1 \wedge$ & برودى \\
\hline .1 .9 &.$- / \mu$ &.$- / 1 f$ & كومهرتز \\
\hline .1 .10 &.$-|r|$ & $-\cdot / r$. & لجستيك \\
\hline
\end{tabular}

مقادير جذر ميانخين مربعات خطا و ميانگين قدر مطلق خطا

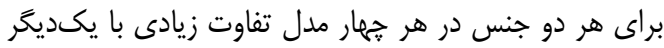

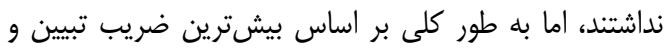

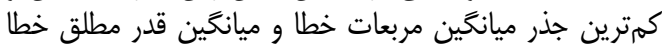

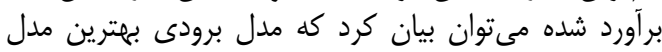

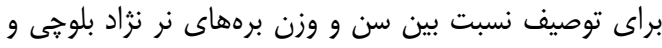

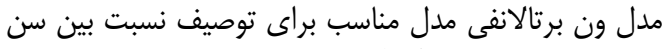

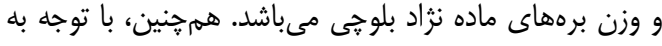

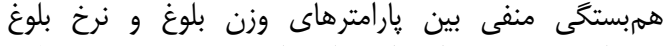

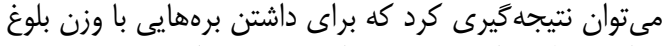

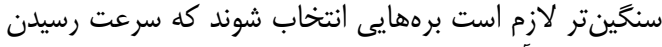
به سن بلوغ آنها كندتر مىباشد برهابي انتخا

$$
\text { تشكر و قدردانى }
$$

اين تحقيق با حمايت مالى دانشعاه آزاد اسلامى واحد

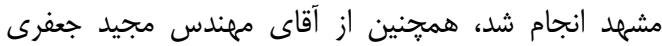

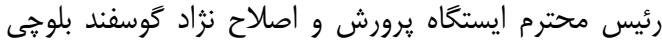
عباسآباد به خاطر در اختيار قرار دادن ركوردهاه

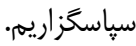

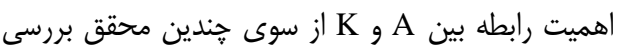

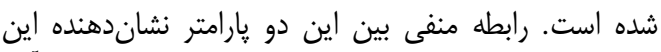

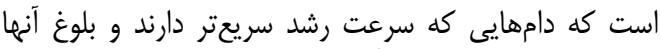

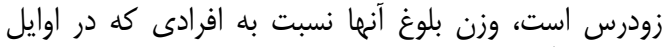

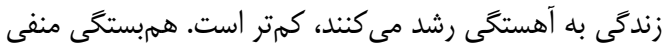

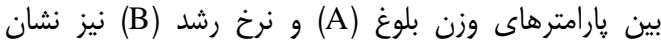

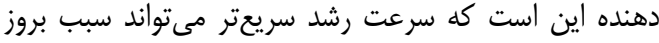

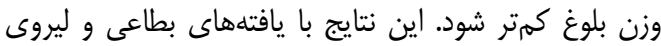

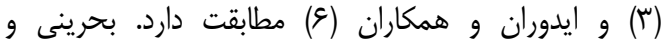

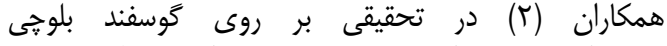

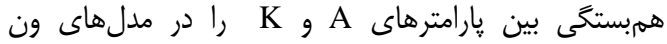

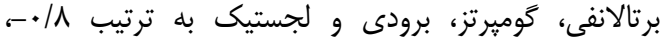
هم /VD همكاران (V) همبستخى بين يارامترهاى A و

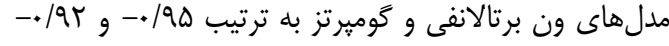

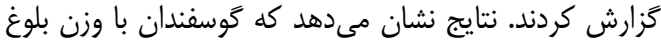
كم تر، سريعتر بالغ مى شعودن

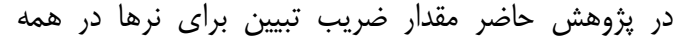

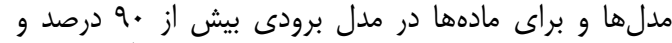

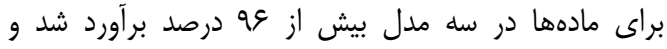


. Arango, J.A. and L.D. Van Vleck. 2002. Size of beef cows; early ideas new developments. Genetic and Molecular Research, 1: 51-63.

2. Bahreini Behzadi, M.R., A.A. Aslaminejad, A.R. Sharifi and H. Simianer. 2014. Comparison of mathematical models for describing the growth of Baluchi sheep. Journal of Agricultural Science and Technology, 14: 57-68.

3. Bathaei, S.S. and P.L. Leroy. 1998. Genetic and phenotypic aspects of the growth curve characteristics in Mehraban Iranian fat-tailed sheep. Small Ruminant Research, 29: 261-269.

4. Bertalanffy, L.V. 1957. Quantitative laws in metabolism and growth. Quarterly Review of Biology, 32: 217-231.

5. Brody, S. 1945. Bioenergetic and Growth. Reinhold Publishing Co, New York, USA, 1023 pp.

6. Eyduran, E., M. Kucuk, K. Karakus and T. Ozedemir. 2008. New Approaches to determination of the best nonlinear function describing growth at early phases of kivircik and morkaraman breeds. Journal of Animal and Veterinary Advance, 7: 799-804.

7. Gbangboche, A.B., T. I. Alkoiret, S. Salifou, F. Farnir, P.L. Leroyand and F.A. Abiola. 2011. Growth pattern of purebred West African dwarf sheep and its crosses with the West African long legged. Research Journal of Animal Science, 5: 6-13.

8. Gompertz, B. 1825. On the nature of the fucntion of the law of human mortality and a new mode of determining the value of live contingencies. Philosophical Transactions of the Royal Society, 182: 513-585.

9. Keskin, I., B. Dag, V. Sariyel and M. Gokmen. 2009. Estimation of growth curve parameters in konya merino sheep. South African Journal of Animal Science, 39: 163-168.

10. Kucuk, M., E. Eyduran. 2009. The Determination of the Best Growth Model for Akkaraman and German Blackheaded Mutton x Akkaraman $b_{1}$ Crosbreed Lambs. Bulgarian Journal of Agricultural Science, 15: 90-92.

11. Kum, D., K. Karakus and T. Ozdemir. 2010. The best non-linear function for body weight at early phase of norduz female lambs. Trakia Journal of Science, 8: 62-67.

12. Lambe, N.R., E.A. Navajas, G. Simm and L. Bünger. 2006. A genetic investigation of various growth models to describe growth of lambs of two contrasting breeds. Journal of Animal Science, 84: 26422654.

13. Najafy, M., G. Rahimi, Z. Ansari, H. Baneh and Z. Yosefi. 2011. Effects of environmental factors on phenotypic growth traits in naini sheep. Research on Animal Production, 2: 12-22 (In Persian).

14. Saghi, D.A., A. Aslaminejad, M. Tahmoorespur, H. Farhangfar, M. Nassiri and G.R. Dashab. 2012. Estimation of genetic parameters for growth traits in baluchi sheep using gompertz growth curve function. Indian Journal of Animal Sciences, 82: 889-892.

15. SAS. 2005. Statistics analysis system user's guide. Release 9.1. SAS Institute Inc., Cary, North Carolina, USA.

16. Tariq, M.M., M.A. Bajwa, A. Waheed, E. Eyduran, F. Abbas, F.A. Bokhari and A. Akbar. 2011. Growth curve in Mengali sheep breed of Balochistan. Journal of Animal \& Plant Science, 21: 5-7.

17. Topal, M., M. Ozdemir, V. Aksakal, N. Yildiz and U. Dogru. 2004. Determination of the best nonlinear function in order to estimate growth in morkaraman and awassi lambs. Small Ruminant Research, 55: 229-232.

18. Ulutas, Z., M. Sezer, Y. Aksoy, E. Sirin, U. Sen, M. Kuran and Y. Akbas. 2010. The effect of birth type on growth curve parameters of karayaka lamb. Journal of Animal and Veterinary Advance, 9: 1384-1388.

19. Verhulst, P.F. 1838. Notice Sur La Loi que la population suit dans son accroissement Correspondence Mathematique et Physique, 10: 113-121.

20. Zendedel Dalir Haghighat, M., A.A. Shadparvar, M. Golshani and M. Naserani1. 2015. Estimation of genetic parameters for growth traits in Guilan Province Sheeps. Research on Animal Production, 6: 126-132 (In Persian). 


\title{
Description of Growth Curve in Male and Female Lambs of Baluchi Breed by Application of Nonlinear Growth Models
}

\author{
Mojtaba Hosseinpour Mashhadi ${ }^{1}$, Mehdi Elahi Torshizi ${ }^{2}$ and Shahab Ehtesham Gharaee ${ }^{3}$ \\ 1- Assistant Professor, Department of Animal Science, Mashhad Branch, Islamic Azad University, Mashhad, Iran, \\ (Corresponding author: mojtaba_h_m@yahoo.com) \\ 2- Assistant Professor, Department of Animal Science, Mashhad Branch, Islamic Azad University, Mashhad, Iran \\ 3- Ph.D. Student, Department of Animal Science, International Ferdowsi University, Mashhad, Iran \\ Received: October 27, 2014 \\ Accepted: February 4, 2015
}

\begin{abstract}
The purpose of this study was to describe growth curve in Baluchi sheep by application of nonlinear growth models. The weight records of 1228 and 676 Baluchi male and female lambs for birth weight, one-month, two-month, three-month, four-month, six-month, nine-month and one year of age were studied. These data had been collected by Baluchi Sheep Breeding Center in Mashhad (Abbas Abad) during 2004 to 2009. Brody, Von Bertalanffy, Gompertz and Logistic nonlinear models were fitted to describe the growth curve. The most suitable model

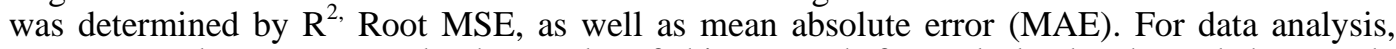
NLIN procedures were used. The results of this research for male lambs showed that Brody model with $\mathrm{R}^{2}$ (90.42\%), Root MSE (8.35) and mean difference between expected and observed value (MAE) (5.65) was the best model compared with others, this value for proper model (Von Bertalanffy) in female lamb were $96.85 \%\left(\mathrm{R}^{2}\right), 4.54$ (Root MSE) and 3.16 (mean difference between expected and observed value). The correlation between $\mathrm{A}$ and $\mathrm{K}$ parameters for Von Bertalanffy, Brody, Gompertz and Logistic were $-0.36,-0.54,-0.3$ and -0.21 , respectively.
\end{abstract}

Keywords: Brody, Gompertz, Logistic, Sheep, Von Bertalanffy 